\title{
Iontronic control of GalnAsP photonic crystal nanolaser
}

\author{
Yoshito Saijo, Keisuke Watanabe, Takumi Watanabe, Yu Terada, Yoshiaki Nishijima, \\ and Toshihiko Babaa)
}

\author{
AFFILIATIONS \\ Department of Electrical and Computer Engineering, Yokohama National University, \\ 79-5 Tokiwadai, Hodogaya-ku, Yokohama 240-8501, Japan
}

a) Author to whom corresponding should be addressed: baba-toshihiko-zm@ynu.ac.jp

\section{ABSTRACT}

In this study, we fabricated a photoelectrochemical circuit using GaInAsP photonic crystal nanolasers as a working electrode. Then, we controlled emission intensity and lasing wavelength of these nanolasers by applying a bias voltage in an ionic solution. The electrochemical working points for the emission intensity and wavelength were observed for the backward and forward biases, respectively. We confirmed that the emission intensity is primarily changed by the surface recombination, which is enhanced by the Schottky barrier near the solid-liquid interface. The wavelength shift is also assumed to be caused by the Pockels effect in the electric double layer of the solution. This control method can maximize and stabilize the performance of photonic bio-chemical sensors and also become an option in controlling the laser diode characteristics.

Micro-scale chemical sensor devices are commonly used in medical diagnoses, bioscience, and environmental monitoring. Ion-sensitive field-effect transistors (ISFETs) $)^{1,2}$ and surface plasmon resonance (SPR) sensors ${ }^{3,4}$ are popular electronic and photonic devices, respectively. Herein, a GaInAsP semiconductor photonic crystal (PC) nanolaser ${ }^{5-7}$ (FIG. 1(a)) has been investigated as another photonic device for detecting chemicals, biomolecules, living cells, and environmental toxins. ${ }^{8-11}$ In the first stage, this nanolaser was used as the sensor to detect the refractive index of the contact medium from optical resonance shifts similar to the SPR sensors. Afterwards, however, we observed that the nanolaser is sensitive to the surface electric charge ${ }^{12,13}$ similar to the ISFET sensors. We call this sensor as an iontronic-photonic sensor.

For example, ion sensitivity occurs in the change of the emission intensity when the nanolaser is immersed in various $\mathrm{pH}$ solutions. ${ }^{12}$ This phenomenon can be explained using the nonradiative surface recombination, which is enhanced by the Schottky barrier height at the semiconductor-solution interface (FIG. 1(b)). The barrier height $e \Delta U$ is modified by the acid dissociation equilibrium of the device surface and the change in the redox potential of ions. This principle has been supported by the emission lifetime measurement ${ }^{12}$ and applied to detect a biomarker from human blood without using any type of spectral analyses. ${ }^{14}$ We also observed a wavelength shift with time when we exposed the device to plasma and successively operated in water. ${ }^{13}$ This shift corresponded well to the evolution of the flatband potential $e U_{\mathrm{fb}}$ at the semiconductor surface, which was measured electrochemically. The result indicates that the wavelength shift was also dependent on the surface charge and emerged from the plasma effect of photo-excited carriers, which were captured at the Schottky barrier.

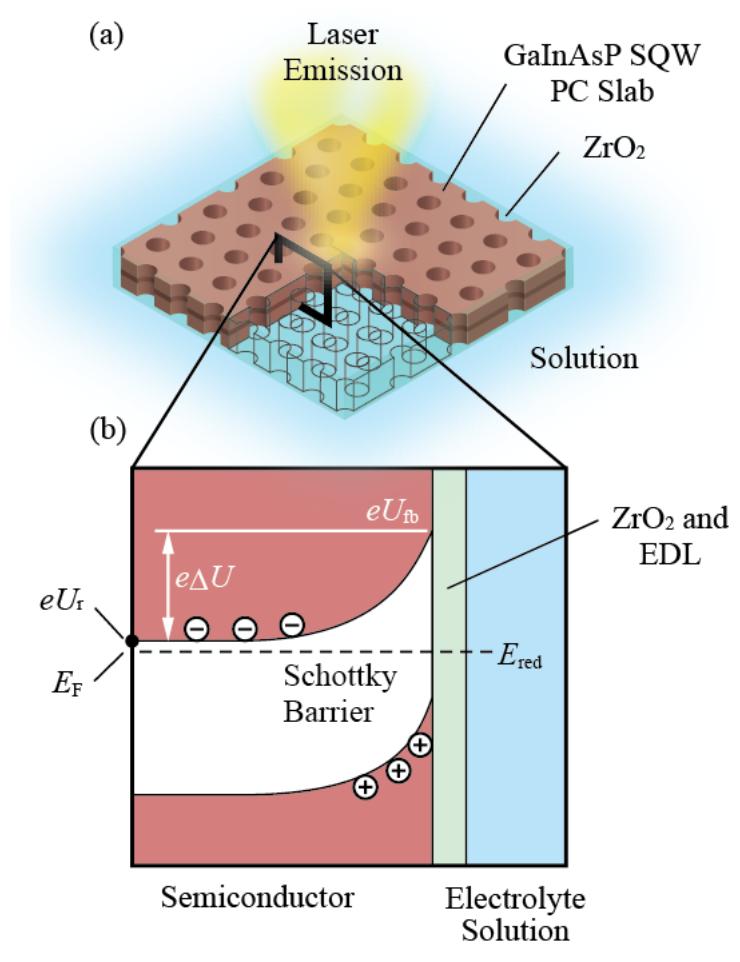

FIG. 1. GalnAsP PC nanolaser. (a) Schematic of the nanolaser in a solution. (b) Schematic of the electronic band near the solidliquid interface.

Meanwhile, the $\mathrm{pH}$ dependence of the emission intensity of nitride semiconductor nanowires in a solution has been reported to be dependent on bias voltage. ${ }^{15}$ The $\mathrm{pH}$ dependency might occur due to the surface recombination modified by $e \Delta U$, similar to that in the nanolaser. Here the rest potential of $e U_{\mathrm{r}}$ corresponding to the conduction band edge in the semiconductor bulk is modulated by the bias 
voltage to ensure that the $\mathrm{pH}$ dependency is maximized. In this study, we attempted to modulate $e \Delta U$ of the nanolaser by developing a photo- electrochemical circuit. We observed that the emission intensity and the wavelength can be controlled by the applied voltage and that their working points are not equal, suggesting the involvement of various principles.

(a)

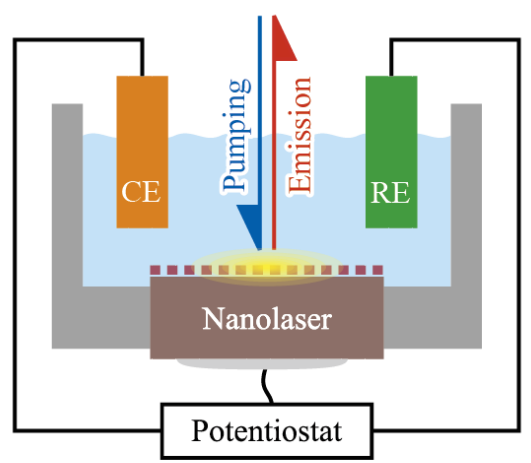

(b)

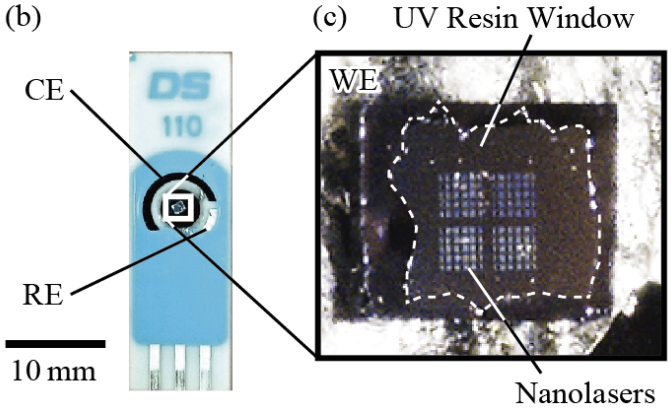

FIG. 2. Photoelectrochemical circuit with nanolasers as a working electrode (WE). (a) Schematic of the electromechanical circuit. (b) Screen print cell used as the circuit. (c) Magnified view of WE at which the nanolaser was soldered.

Details of the structure, fabrication process, and measurement method of the nanolaser are the same as those in Ref. 7. The H0-type nanocavity consisting of 2-4 shifted holes in a triangular lattice hole array PC slab was formed into a weak n-type GaInAsP single quantum well (SQW) epiwafer. It is operated at a wavelength $\lambda \approx 1.55 \mu \mathrm{m}$ by 0.98 $\mu \mathrm{m}$ photopumping at room temperature. The extraction of light output was enhanced by modulating the diameter of the holes around the cavity. ${ }^{16}$ The nanolaser is permanently operated for more than a day in a solution, including ions, by coating the device with $\sim 3 \mathrm{~nm} \mathrm{ZrO}_{2}$ using an atomic layer deposition. ${ }^{12}$ We employed an electrochemical circuit with standard three electrode type, namely, working electrode (WE), counter electrode (CE), and reference electrode (RE), ${ }^{17}$ (FIG. 2(a)). Specifically, we used a screen print cell (DropSens, 110) (FIG. 2(b)) for the circuit. A 1 mm square nanolaser chip was cleaved out and its back surface was soldered to the WE with indium. Then, the surface apart from the nanolasers was insulated by the UV curable resin.
The CE was carbon (C) and the quasi RE was Ag. We used a $10 \mathrm{mM} \mathrm{K}_{2} \mathrm{SO}_{4}$ for the ionic solution, and $\mathrm{H}_{2} \mathrm{SO}_{4}$ or $\mathrm{KOH}$, as the $\mathrm{pH}$ element. To precisely control $\mathrm{pH}$ particularly around 7 , buffer elements should be included. But we did not use it to avoid the complication of the phenomena. Considering that $\mathrm{K}_{2} \mathrm{SO}_{4}$ is inactive to the $\mathrm{Ag}$ electrode, the electric potential is stabilized, and its reference potential can be calibrated using the saturated $\mathrm{Ag} / \mathrm{AgCl}$ standard electrode. An electro- chemical analyzer (CH Instruments, ALS-650E) was utilized as potentiostat. As shown in FIG. 1(b), $\Delta U$ is given by $U_{\mathrm{r}}-U_{\mathrm{fb}}$, where $U_{\mathrm{fb}}$ and $U_{\mathrm{r}}$ are obtained using MottSchottky plot and open-circuit measurements, respectively. In the open-circuit measurement, a white light from a lightemitting diode was irradiated to simulate the nanolaser photopumping condition. In the nanolaser operation, the pump light was irradiated while applying the bias using an amperometric technique.

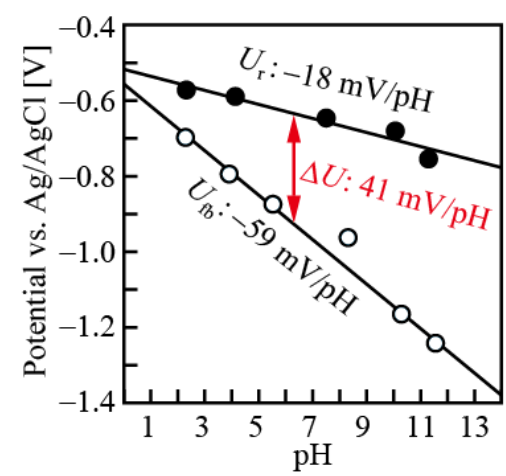

FIG. 3. Electrochemically measured $\mathrm{pH}$ dependencies of $U_{\mathrm{fb}}$ and $U_{r}$ of the nanolaser, which include an error of $<0.03 \mathrm{~V}$.

Figure 3 shows the $\mathrm{pH}$ dependency of $U_{\mathrm{fb}}$ and $U_{\mathrm{r}}$. Here, $U_{\mathrm{fb}}$ agreed well with the Nernst response of $-59.2 \mathrm{mV} / \mathrm{pH},{ }^{17}$ whereas $U_{\mathrm{r}}$ has low value (i.e., $-18 \mathrm{mV} / \mathrm{pH}$ ), resulting in the $\Delta U$ response of $41 \mathrm{mV} / \mathrm{pH}$. If the equilibrium were maintained between the Fermi energy and redox potential through the transportation of electrons from ions in the solution to the semiconductor, $U_{\mathrm{r}}$ would also agree with the Nernst response, and $\Delta U$ would not depend on $\mathrm{pH}$. Actually, however, the equilibrium was hindered, and the $U_{\mathrm{r}}$ response was reduced in the presence of $\mathrm{ZrO}_{2}$ film.

Based on the result displayed in FIG. 3, we estimated the change of the electronic band (FIG. 4). Considering the Nernst response and saturated $\mathrm{Ag} / \mathrm{AgCl}$ electrode measurement, the redox potential of $\mathrm{H}^{+}$is $-0.61 \mathrm{~V}$ at $\mathrm{pH}=$ 7. Therefore, the bias voltage $V$ of this value is equivalent to the equilibrium condition that has no bias. Then, the Schottky barrier of $\Delta U \approx 0.3 \mathrm{~V}$ is formed. When the bias voltage is more positive than $-0.61 \mathrm{~V}$, the electric field is applied mainly to the barrier on a reverse bias condition. When it is more negative than $-0.61 \mathrm{~V}$, the barrier is reduced because of the forward bias condition, and electrons are 
accumulated near the semiconductor- $\mathrm{ZrO}_{2}$ interface. Here, a small current is leaked through $\mathrm{ZrO}_{2}$ into the solution, and the electric field is mainly applied to the electric double layer (EDL) formed against the counter ions diffused in the solution.
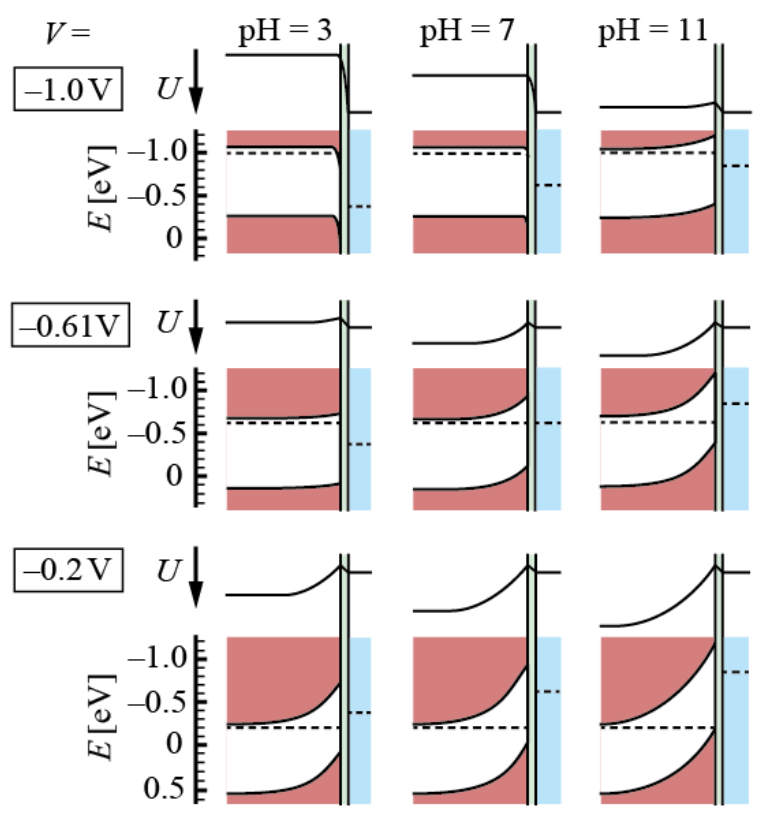

FIG. 4. Band energy $E$ and electric potential $U$, which depend on $\mathrm{pH}$ and bias voltage $\mathrm{V}(\mathrm{vs} . \mathrm{Ag} / \mathrm{AgCl})$. The band is presented similar to FIG. 1(b). The dotted lines indicate $E_{\mathrm{F}}$ and $E_{\text {red. }}$. (a)

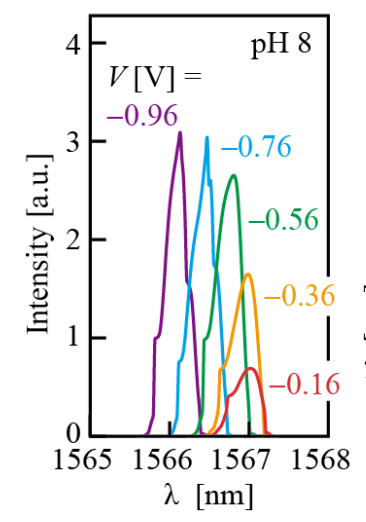

(b)

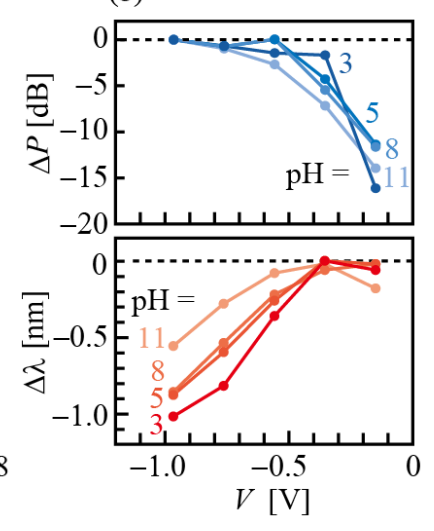

FIG. 5. Emission intensity and lasing wavelength regulation of the nanolaser by the bias voltage $V$ (vs. Ag/AgCl). (a) Lasing spectrum, where the measurement resolution was $0.2 \mathrm{~nm}$. (b) Variation of the emission intensity $\Delta P$ and the lasing wavelength $\Delta \lambda$ with $V$ at various $\mathrm{pH}$ conditions, which include fluctuations of $<1 \mathrm{~dB}$ and $<0.3$ $\mathrm{nm}$, respectively.

Then, we performed the regulation of the emission intensity and lasing wavelength (FIG. 5; we repeated the same experiment several times and observed almost the same results.). As observed in the figure, the emission intensity $P$ and the wavelength $\lambda$ change on the reverse and forward bias sides, respectively, although fine behaviors might include some fluctuations due to unstable $\mathrm{pH}$ particular at 5-8 in the absence of buffer elements. In the region where this change occurred, the response $\Delta P / \Delta V$ was from -75 to $-20 \mathrm{~dB} / \mathrm{V}$ and $\Delta \lambda / \Delta V$ was $1-2 \mathrm{~nm} / \mathrm{V}$. Here, we define the $\mathrm{pH}$ sensitivity $S_{\mathrm{pH}}$ to $P$ as

$$
S_{\mathrm{pH}}=(\Delta P / \Delta V) \cdot(\Delta U / \Delta \mathrm{pH}) .
$$

On the reverse bias side, $S_{\mathrm{pH}}$ is from -0.78 to $-2.93 \mathrm{~dB} / \mathrm{pH}$. These values are slightly larger than the experimental values from -0.3 to $-1.6 \mathrm{~dB} / \mathrm{pH}$ in Ref. 12 . The possible reason is the low pump level against the lasing threshold in this experiment, which enhances the surface recombination. $P$ decreased by more than $10 \mathrm{~dB}$ at $V \approx-0.2 \mathrm{~V}$, and the laser operation stopped beyond because of the remarkable surface recombination by large $\Delta U$. The electrochemical working point for the maximum $\mathrm{pH}$ dependency was $-0.4 \mathrm{~V}$.

Contrastingly, $\lambda$ exhibited a $1 \mathrm{~nm}$ blueshift at a maximum on the forward bias side, with the electrochemical working point of $-0.8 \mathrm{~V}$. The different working points between $P$ and $\lambda$ indicate different principles. Several factors can be considered for the wavelength shift, but those based on $\Delta U$ cannot explain in any cases. This result does not agree with the conclusion in Ref. 13, wherein excited carriers are trapped in the optical confinement layers outside of the SQW active layer by $\Delta U$ and the wavelength becomes shorter because of the carrier plasma effect. ${ }^{13}$ We also considered the carrier plasma effect of electrons accumulated at the interface by the forward bias. However, this effect was estimated to be minimal because the reduction in the SQW's refractive index due to this effect is of 0.001 order and the confinement factor of the laser mode into the SQW was calculated to be $2.1 \%$. The band filling effect is another carrier effect, which produces a 0.1 order reduction in the SQW's index near the electronic band edge wavelength. ${ }^{18}$ Such a large index change could be a reason for the wavelength shift even with the small confinement factor. Nonuniform in-plane distributions of electrons and holes caused by the bias could modify the modal index and shift the wavelength. In this case, however, the wavelength shift should be accompanied by the emission intensity change, which does not agree with the observed behaviors. A reasonable explanation we found is the Pockels effect at the EDL of water. ${ }^{19}$ The reported Pockels coefficient $r=200$ $300 \mathrm{pm} / \mathrm{V}$ is much larger than $\sim 10 \mathrm{pm} / \mathrm{V}$ of $\mathrm{LiNbO}_{3}$. Moreover, the index change $\Delta n$ is expressed as

$$
\Delta n=-\frac{1}{2} n_{w}{ }^{3} r E_{\mathrm{EDL}}=-\frac{n_{w}{ }^{3} r V_{\mathrm{EDL}}}{2 \lambda_{\mathrm{D}}},
$$

where $V_{\mathrm{EDL}}$ is the potential difference at the EDL and $\lambda_{\mathrm{D}}$ is 
the Debye length. We assume that the index of water, $n_{\mathrm{w}}=$ 1.321 , and $\lambda_{\mathrm{D}}=1.76 \mathrm{~nm}$ for $10 \mathrm{mM} \mathrm{K}_{2} \mathrm{SO}_{4}$, and obtain a large $\Delta n / \Delta V_{\mathrm{EDL}}=0.13-0.20 / \mathrm{V}$. The wavelength shift was calculated to be $0.9-1.4 \mathrm{~nm} / \mathrm{V}$ from the overlap of the EDL with the laser mode obtained through the finite-difference time-domain simulation. At $\mathrm{pH}=3$ and $V=-1 \mathrm{~V}, \Delta V_{\mathrm{EDL}}$ at the forward bias is higher than $0.7 \mathrm{~V}$. Hence, the expected shift of $0.6-0.9 \mathrm{~nm}$ agrees with the experimental value in FIG. 5(b). The penetration depth of the laser mode into the solution is $110-140 \mathrm{~nm},{ }^{5}$ which is much longer than the assumed $\lambda_{\mathrm{D}}$. Therefore, even though $\mathrm{K}_{2} \mathrm{SO}_{4}$ concentration changes by more than two digits, $\Delta \lambda$ does not change significantly because the increase in $\lambda_{\mathrm{D}}$ reduces $\Delta n$ while increasing the modal overlap and vice versa.

In conclusion, we constructed the photoelectrochemical circuit using GaInAsP PC nanolasers and then demonstrated the iontronic control of their emission intensity and lasing wavelength by applying the bias voltage. The different working points allow independent control of the intensity and wavelength, which can be an option for externally controlling the laser diodes. The bias voltage available is limited in the range from $-0.1 \mathrm{~V}$ to $-1.0 \mathrm{~V}$ due to the stop of the laser operation on the reverse bias side and the enhanced leakage current through $\mathrm{ZrO}_{2}$ film on the forward bias side, respectively. The emission intensity can be widely changed, while the control of the wavelength may be limited to around $1 \mathrm{~nm}$. We assume that such bias can improve the sensitivity and robustness of the nanolaser biochemical sensors, because the ultrahigh sensitivity to biomolecules, which has been observed so far, ${ }^{13}$ is considered to rely on the delicate initial charge of the device and the charge transfer from adsorbed bio-molecules. Moreover, investigating similar electrochemical effects in other photonic sensors is also interesting. As discussed in Ref. 13, unexpected sensing behaviors have been reported for many other semiconductor photonic sensors although these effects have not been discussed explicitly.

\section{Acknowledgments}

This work was supported by Grant-in-Aid \#16H06334 from the Ministry of Education Culture, Sports, Science and Technology, Japan.

\section{References}

1 A. Kim, C. S. Ah, H. Y. Yu, J. H. Yang, I. B. Baek, C. G. Ahn, C. W. Park, and M. A. Jun, Appl. Phys. Lett. 91, 103901 (2007).

2 C. W. Liao, J. C. Chou, T. P. Sun, S. K. Hsiung, and J. H. Hsieh, J. Electrochem. Soc. 156, 2 (2009).

3 A. J. Haes, W. P. Hall, L. Chang, W. L. Klein, and R. P. Van Duyne, Nano Lett. 4, 6 (2004).

4 J. Lu, D. Spaic, F. Delport, T. V. Stappen, I. Detrez, D. Daems, S. Vermeire, A. Gils, and J. Lammertyn, Analytical Chem. 89,
3664 (2017).

5 O. Painter, R. K. Lee, A. Scherer, A. Yariv, J. D. O'Brien, P. D. Dapkus, and I. Kim, Science 284, 1819 (1999).

6 H. Y. Ryu, S. H. Kwon, Y. J. Lee, Y. H. Lee, and J.-S. Kim, Appl. Phys. Lett. 80, 3476 ( 2002).

7 S. Kita, K. Nozaki, S. Hachuda, H. Watanabe, Y. Saito, S. Otsuka, T. Nakada, Y. Arita, and T. Baba, IEEE J. Sel. Top. Quantum Electron. 17, 1632 (2011).

8 M. Loncar, A. Scherer, and Y. Qiu, Appl. Phys. Lett. 82, 4648 (2003).

9 S. Hachuda, T. Watanabe, D. Takahashi, and T. Baba, Opt. Express 24, 12886 (2016).

10 H. Abe, M. Narimatsu, T. Watanabe, T. Furumoto, Y. Yokouchi, Y. Nishijima, S. Kita, A. Tomitaka, S. Ota, Y. Takemura, and T. Baba, Opt. Express 23, 17056 (2015).

11 D. Takahashi, S. Hachuda, T. Watanabe, Y. Nishijima, and T. Baba, Appl. Phys. Lett. 106, 131112 (2015).

12 K. Watanabe, Y. Kishi, S. Hachuda, T. Watanabe, M. Sakemoto, Y. Nishijima, and T. Baba, Appl. Phys. Lett. 106, 021106 (2015).

13 T. Watanabe, Y. Saijo, Y. Hasegawa, K. Watanabe, Y. Nnishijima, and T. Baba, Opt. Express. 5, 24469 (2017).

14 K. Watanabe, M. Nomoto, F. Nakamura, S. Hachuda, A. Sakata, T. Watanabe, Y. Goshima, and T. Baba, Biosen. Bioelectron. 117, 161 (2018).

15 J. Wallys, J. Teubert, F. Furmayr, D. M. Hofmann, and M. Erickhoff, Nano Lett. 12, 6081 (2012).

16 M. Narimatsu, S. Kita, H. Abe, and T. Baba, Appl. Phys. Lett. 100, 121117 (2012).

17 A. J. Bard, and L. R. Faulkner, Electrochemical methods: Fundamentals and Applications 2nd ed., Wiley (2000).

18 B. R. Bennett, R. A. Soref and J. A. Del Alamo, IEEE J. Quantum Electron. 26, 113 (1990).

19 Y. Nosaka, M. Hirabayashi, T. Kobayashi, and E. Tokunaga, Phys. Rev. B 77, 241401 (2008). 